\title{
High-Performance Liquid Chromatographic Method for Separation and Quantification of Intact Glucosinolates
}

by N. Kaushik* / A. Agnihotri

published in Vol. 49, 281-284 (1999)

Table I on page 282 was incomplete. Please find below the correct version of this Table.

Table I. Major glucosinolates of different Brassica species.

\begin{tabular}{|c|c|c|c|c|}
\hline No. & Systematic name & Common name & Structure of R group in Figure 1 & Found in species: \\
\hline 1 & Allyl glucosinolates & Sinigrin & $\mathrm{CH}_{2}=\mathrm{CH}-\mathrm{CH}_{2-}$ & $\begin{array}{l}\text { B. juncea, } B . \text { nigra, } \\
B . \text { carinata, } B . \text { oleracea }\end{array}$ \\
\hline 2 & 3-Butenyl glucosinolate & Gluconapin & $\mathrm{CH}_{2}=\mathrm{CH}-\mathrm{CH}_{2} \mathrm{CH}_{2}-$ & B. campestris, B. juncea \\
\hline 3 & $\begin{array}{l}\text { 2-Hydroxy-3-butenyl } \\
\text { glucosinolates }\end{array}$ & Progoitrin & $\mathrm{CH}_{2}=\mathrm{CH}-\mathrm{CHOH}-\mathrm{CH}_{2}-$ & B. napus, B. oleracea \\
\hline 4 & 4-Pentenyl glucosinolates & Glucobrassicanapin & $\mathrm{CH}_{2}=\mathrm{CH}-\left(\mathrm{CH}_{2}\right)_{3}-$ & $\begin{array}{l}\text { Minor glucosinolate in all } \\
\text { Brassica species }\end{array}$ \\
\hline 5 & $\begin{array}{l}\text { 2-Hydroxy-4-pentenyl } \\
\text { glucosinolates }\end{array}$ & Napoleiferin & $\mathrm{CH}_{2}=\mathrm{CH}-\mathrm{CH}_{2}-\mathrm{CHOH}-\mathrm{CH}_{2}-$ & $\begin{array}{l}\text { Minor glucosinolate in all } \\
\text { Brassica species }\end{array}$ \\
\hline 6 & 3-Indolylmethyl glucosinolate & Glucobrassicin & & $\begin{array}{l}\text { Minor glucosinolate in all } \\
\text { Brassica } \text { species }\end{array}$ \\
\hline 7 & $\begin{array}{l}\text { 4-Hydroxy-3-indolylmethyl } \\
\text { glucosinolates }\end{array}$ & 4-Hydroxyglucobrassicin & & $\begin{array}{l}\text { Minor glucosinolate in all } \\
\text { Brassica species }\end{array}$ \\
\hline
\end{tabular}

